\title{
A concept of cost-saving space system designed for the detection of NEOs (EKOZONT)
}

\author{
Andrey Shugarov ${ }^{\mathrm{a}}$, Igor Savanov, and Boris Shustov \\ Institute of Astronomy of RAS, Moscow, Russia
}

\begin{abstract}
In recent years many countries put effort to build systems to detect and to monitor near Earth objects (NEOs) with a size down to $100 \mathrm{~m}$ that could lead to catastrophic results in case of collision with the Earth. EKOZONT is a concept of cost-effective space system designed for the detection of NEOs down to size of $100 \mathrm{~m}$ not later than 1525 days before the possible collision with the Earth.
\end{abstract}

\section{Scientific goals}

In recent years a number of projects of systems to detecting and monitoring near Earth objects (NEOs) of size down to $100 \mathrm{~m}$ were discussed. There are several variants of NEOs detection systems:

- several large ground-based wide field optical telescopes located in different hemispheres of the Earth;

- space system with optical and/or infrared telescopes.

In this paper we consider the space-based cost-effective optical range system. The proposed system can be used for detection and monitoring of space debris also. Scientific objectives that can be studied simultaneously with the search of NEOs are:

- dynamics of Solar System bodies;

- supernova, variable stars;

- stellar activity.

\section{EKOZONT project}

For NEOs detection system we propose the VT-77 telescope [1] with $0.75 \mathrm{~m}$ aperture, very large field of view and high image quality. Telescopes are designed for operation in direct pointing mode.

The small-size space platform manufactured by Lavochkin Association will be used as a spacebus for the wide field telescope. Distinctive features of the platform are the subarcsecond accuracy of stabilization, fast settling time, the possibility of very frequent repointing.

The spacecraft will operate at a low orbit. The main advantages of the selected orbit are in the low launch cost and high speed of radio link. It is supposed to launch two identical spacecrafts with a single launch vehicle "Soyuz" with the upper stage "Fregat". In addition to the main telescope an auxiliary $40 \mathrm{~cm}$ telescope can be accommodated on the bus. The additional small telescopeshould have very large field of view (10 deg) with time of a complete all-sky survey of about one day.

The system with two types of telescopes is "dual-channel":

- $40 \mathrm{~cm}$ telescope will be effective to discover the nearest bright NEOs and space debris;

- the main telescope will be focused to search the weakest and the most distant NEOs.

a e-mail: shugarov@inasan.ru

This is an Open Access article distributed under the terms of the Creative Commons Attribution License 4.0, which permits unrestricted use, distribution, and reproduction in any medium, provided the original work is properly cited. 


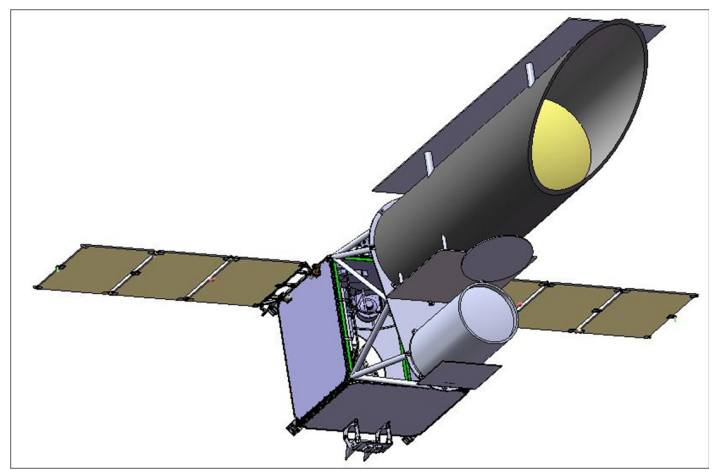

Fig. 1. Spacecraft layout with telescopes VT-77 and auxiliary $40 \mathrm{~cm}$ telescope.

To determine the area of NEOs detection for space telescope and to determine the $\mathrm{S} / \mathrm{N}$ ratio within it we perform a simulation. The VT-77 telescope with $0.75 \mathrm{~m}$ aperture and 7 deg field of view is efficient for detecting $100 \mathrm{~m}$ NEOs not later than 15-25 days before possible collision. For $50 \mathrm{~m}$ bodies larger instrument is required. The additional small telescope with a very large field of view (10 deg) will be efficient to discover the nearest bright NEOs and space debris.

\section{References}

1. V.Yu. Terebizh, Astronomische Nachrichten 332, (2011) 714. 